\title{
5. Rezensionsabhandlungen
}

Dresdner Schriften zum Bürgerentscheid, hrsg. v. Peter Neumann, Deutsches Institut für Sachunmittelbare Demokratie an der Technischen Universität Dresden (DISUD). Nomos Verlagsgesellschaft Baden-Baden:

Kerstin Müller: Bürgerbegehren und Bürgerentscheid in Delmenhorst - 1996 bis 2006. Dresdner Schriften zum Bürgerentscheid Bd. 1, 2010, 76 S., 19 Euro.

Jörn Zylla: Bürgerbegehren und Bürgerentscheid in Dresden - 1992 bis 2009. Dresdner Schriften zum Bürgerentscheid Bd. 2, 2010, 140 S., 34 Euro.

Die kommunale Ebene bietet für direkte Demokratie mittlerweile ein breites Erfahrungsfeld. Nachdem Baden-Württemberg über drei Jahrzehnte damit allein stand, folgten zwischen 1990 und 2005 alle anderen Bundesländer und ermöglichten Bürgerbegehren und -entscheide zu kommunalpolitischen Fragen. Die unterschiedlichen und teilweise unnötig hemmenden Verfahrensregeln machen wissenschaftliche Begleitung sowohl der Praxis als auch der Gesetzgebung zur lohnenden Aufgabe.

In enger Kooperation bemühen sich der Verein Mehr Demokratie und die Forschungsstellen Bürgerbeteiligung in Marburg und Wuppertal um Erfassung der Daten und deren Auswertung durch Bürgerentscheidsberichte, Merkblätter, Empfehlungen für die Gesetzgebung und weitere Veröffentlichungen. Aktivität auf diesem Felde entfaltet auch das von Peter Neumann geführte Deutsche Institut für Sachunmittelbare Demokratie (DISUD) an der Technischen Universität Dresden. Neben den „Studien zur Sachunmittelbaren Demokratie“ mit Habilitationsschriften sowie qualifizierten Dissertationen und Forschungsarbeiten hat Peter Neumann im Nomos-Verlag die hier angezeigte Reihe „Dresdner Schriften zum Bürgerentscheid" begonnen. Sie soll auf bescheidenerem Niveau exemplarische Einblicke in kommunale Praxis gewähren. Denkbar wären sowohl gründliche Einzelfallstudien als auch Untersuchungen zu einzelnen Verfahrensregeln wie Frist und Kostendeckungsvorschlag oder Berichte über sich thematisch häufende Initiativen, z.B. gegen die Schließung oder Privatisierung öffentlicher Einrichtungen oder gegen Einkaufszentren.

Wohl bedingt durch das nur beschränkt verfügbare Datenmaterial und orientiert an Neigungen der Autoren verzichtet die Publikationsreihe indes auf solche inhaltlichen Vorgaben und beschränkt sich stattdessen jeweils auf eine Gemeinde mit mehreren Bürgerbegehren. Die Einbuße an verallgemeinerbaren Aussagen hofft der Herausgeber durch örtliches Interesse an solcher Dokumentation aus- 
zugleichen. Diese Ortsnähe spiegelt sich in freundlichen Vorworten des jeweiligen Stadtoberhaupts.

Kerstin Müller berichtet in ihrem schmalen Bändchen über sieben angezeigte Bürgerbegehren der Jahre 1996 bis 2006 im niedersächsischen Delmenhorst (80.000 Einw.). Diese relativ hohe Zahl erklärt sie mit dem Engagement zweier Personen, die mehrere Verfahren initiierten. Dass solches Engagement in Delmenhorst durchaus auf offene Ohren stößt, belegt die Tatsache, dass zwar kein Verfahren zum Bürgerentscheid führte, aber das Bürgerbegehren in fünf Fällen in der Sache erfolgreich war und der Gemeinderat nur bei zwei unzulässigen Bürgerbegehren in der Sachfrage gegenteilig entschied. Inhaltlich ging es in drei Fällen um die Frage öffentlicher oder privater Trägerschaft (Abfallwirtschaft, Abwasser, Wohnungsbau), des Weiteren um Großmarktansiedlung, Baumschutzsatzung, Badsanierung oder -neubau und Bürgerhaushalt. Die jeweils knappe Darstellung der Fälle folgt einem gleichbleibenden Schema: Hintergrund, Durchführung, Ergebnis. In einer Schlussbetrachtung streift die Autorin noch einzelne gemeinsame Aspekte wie Themeneignung, Rolle der Medien, Öffentlichkeitsarbeit und Akteure, ohne sie allerdings weiter zu vertiefen.

Auf breiteres Interesse dürfte die Schrift von Jörn Zylla über Dresden schon deswegen stoßen, weil sie Verfahren dokumentiert, die zum Teil weltweites Aufsehen erregten, insbesondere Bürgerbegehren und -entscheid zum Thema Waldschlösschenbrücke und Weltkulturerbe „Dresdner Elbtal“. Überörtliche Schlagzeilen machten aber auch Bürgerbegehren zur Autobahntrasse Dresden-Prag und gegen den Standort Großer Garten für VW. Nach einer kurzen Einleitung und einem Abschnitt über Rechtsgrundlagen und Kriterien für direkten oder indirekten Erfolg (durch ausreichende Mehrheit beim Bürgerentscheid oder entsprechenden Sachbeschluss des Stadtrats) schildert der Autor vierzehn Verfahren eingehend, gegliedert in Hintergrund, Inhalt, Unterschriftensammlung, Zulässigkeit und Erfolg.

Zweimal sahen sich ÖTV, Grüne Liga und PDS in den 90er Jahren veranlasst, ihrer Forderung nach ungeschmälertem Erhalt des Straßenbahnnetzes durch ein Bürgerbegehren Nachdruck zu verleihen, und hatten damit in der Sache Erfolg. Das Bürgerbegehren für eine stadtnahe Autobahntrasse wurde dagegen von CDU, FDP/DSU initiiert und gewann den Bürgerentscheid, so dass der Bund seine entsprechende Planung umsetzen konnte.

In der schon seit hundert Jahren virulenten Frage einer Elbbrücke zwischen den Stadtteilen Johannstadt und Radeberger Vorstadt gab es sogar drei Bürgerbegehren. Das erste begann die PDS schon neun Tage vor dem Stadtratsbeschluss 1996 zugunsten der Waldschlösschenbrücke und strebte stattdessen „Zwei Dresden-typische, kürzere und kostengünstigere Brücken“ an, was jedoch vom Verwaltungsgericht als zu unbestimmt und deswegen für unzulässig erklärt wurde. Nach jahrelangen Verzögerungen und Mehrheitsgewinn der Brückengeg- 
ner bei der Kommunalwahl 2004 organisierte ein von CDU, FDP und ADAC initiierter Verein ein Bürgerbegehren zugunsten der Waldschlösschenbrücke, die bis dahin noch unveränderte Beschlusslage also unterstützend, und erzielte im Februar 2005 mit rd. 68\% der Stimmen einen deutlichen Erfolg.

Doch schon im Juli 2004 hatte die UNESCO das Dresdner Elbtal in die Welterbeliste aufgenommen und drohte nunmehr mit Entzug dieses Ansehen und Tourismus fördernden Titels. Der dadurch entfachte Streit zwischen der Brükkengegnermehrheit im Stadtrat und der Aufsichtsbehörde über den Vollzug des Bürgerentscheids ging bis zum Bundesverfassungsgericht, das trotz Nichtannahme der Verfassungsbeschwerde durchblicken ließ, dass der in einer förmlichen Abstimmung festgestellte Bürgerwille Vorrang haben könne. Ein nach Ablauf der dreijährigen Bindungsfrist gestartetes Bürgerbegehren zugunsten eines durchgängigen Tunnels statt der Brücke erzielte zwar eine breite Zustimmung, wurde jedoch von Aufsichtsbehörde und Verwaltungsgerichten wegen Fristablaufs und unzureichenden Kostendeckungsvorschlags als unzulässig zurückgewiesen. Daraufhin entzog die UNESCO den Welterbetitel. Die im Bau befindliche Brücke spaltet die Dresdner Bürgerschaft bis heute.

Während das Bürgerbegehren zum Schutz der Elbwiesen den Stadtrat von einer partiell ins Auge gefassten Bebauung („Laubegast“) Abstand nehmen ließ, also indirekt erfolgreich war, scheiterte das Begehren gegen den Standort Großer Garten für die VW-Autofabrik knapp am Unterschriftenquorum von damals fünf Prozent, bewirkte aber zumindest Verkleinerung und Begrünung. Wie differenziert der Erfolg eines Bürgerbegehrens zu bewerten ist, wird auch deutlich am Bürgerentscheid in der Ortschaft Dresden-Cossebaude, die sich wegen befürchteter Verkehrsbelastung mit großer Mehrheit gegen einen Brückenneubau aussprach, was aber als rechtlich unverbindliche teilörtliche Stellungnahme zu den Straßenbauplänen von Land, Kreis und Stadt den Bau nicht verhindern konnte.

Umstritten war auch die Randbebauung des Neumarkts neben der Frauenkirche. Die Gesellschaft Historischer Neumarkt versuchte im Jahr 2002 mit Hilfe eines Bürgerbegehrens die weitgehende Wiederherstellung nach historischen Maßgaben sicher zu stellen. Diese formulierte sie detailliert in zehn Punkten eingedenk der gerichtlich beanstandeten Bestimmtheitsmängel des ersten Bürgerbegehrens gegen die Waldschlösschenbrücke. Obwohl unter den Vorbehalt rechtlicher Möglichkeit und notwendiger Abwägung gestellt, sahen Stadtverwaltung und Stadtrat in diesen Vorgaben einen Verstoß gegen das Baugesetzbuch. Das über diese Zulässigkeitsfrage angestrengte Verwaltungsgerichtsverfahren ließen beide Seiten ruhen und erreichten über die Art der Bebauung weitgehende Übereinstimmung. Nur beim Gelände des früheren Gewandhauses, das die Gesellschaft unbebaut lassen will, kam es nochmals zum Konflikt, bis der Stadtrat im Jahr 2008 einer diesbezüglichen Bürgerbegehrensinitiative entsprach und von einer Bebauung insoweit absah. 
Mangels ausreichender Unterstützung keinen Erfolg hatte das Bürgerbegehren gegen den Verkauf von 50.000 städtischen Wohnungen, wodurch sich die Stadt im Jahr 2006 komplett von ihren Schulden befreite. Indirekt erfolgreich war dagegen das vorbeugend eingereichte Bürgerbegehren mit dem Ziel, die Krankenhäuser als städtische Eigenbetriebe zu erhalten und nicht (zwecks Tariffreiheit) zu einer $\mathrm{GmbH}$ umzuwandeln.

Zylla gelingt eine spannend zu lesende Dokumentation wichtiger Ausschnitte der Dresdner Kommunalpolitik. Sie bietet Anschauungsmaterial für grundsätzliche Fragen über Rolle, Ausgestaltung und Handhabung des Instruments Bürgerbegehren und -entscheid. Deutlich wird z.B., dass die durch die unglückliche Fristenregelung verursachte Einteilung in initiierende und kassierende Bürgerbegehren die tatsächlichen Vorgänge höchst unzureichend erfasst; denn offenbar gibt es auch vorbeugende Bürgerbegehren und solche, die weitere Schritte verhindern oder Beschlüsse abwandeln oder nach Zweifeln bekräftigen wollen.

Weil Zylla nur empirische Grundlagen liefern will für weitere Untersuchungen, beschränkt er sich zum Schluss auf die Benennung lohnender Fragen wie Veränderung der Sach- oder Rechtslage, Rolle der Parteien und des Gemeinderats, politische Kultur und direkte Demokratie. Das umfangreiche Literatur- und Quellenverzeichnis entspricht der Gründlichkeit seiner soliden Arbeit.

Für Sommer 2011 war ein dritter Band der Dresdner Schriften angekündigt, und zwar von Tilo Hellmann über Bürgerbegehren und Bürgerentscheid im sächsischen Freiberg, ist aber bis Redaktionsschluss nicht erschienen. Es wäre zu wünschen, dass solche Dokumentationen als Grundlage für weitere Untersuchungen im Internet kostenlos zur Verfügung stünden und gelegentlich aktualisiert würden.

Roland Geitmann

Martin Müller: Bürgerbeteiligung in Finanzfragen. Mittelbare und unmittelbare Bürgerbeteiligung im kommunalen Haushaltswesen de lege lata und de lege ferenda, Studien zur sachunmittelbaren Demokratie, Band 5, Nomos Verlagsgesellschaft: Baden-Baden 2009, 299 S., 49,00 Euro.

Die Ressourcenverteilung durch den kommunalen Haushalt ist das wichtigste Mittel zur Steuerung der Kommunalpolitik. Deshalb liegt der Gedanke nahe, den Einfluss der Bürger auf den Haushalt zu verstärken, sei es durch spezielle Instrumente der Beeinflussung der Haushaltsentscheidung der Vertretung, sei es, indem man auf den „Umweg“ über die Vertretung verzichtet und die Bürger selbst entscheiden lässt. Bei keiner anderen kommunalen Entscheidung sind allerdings so viele rechtliche und tatsächliche Restriktionen zu beachten wie beim Haushalt, denn dieser muss selbst rechtmäßig sein und soll der Kommune recht- 
mäßiges wie zweckmäßiges Handeln ermöglichen. Ein Ausgleich von Einnahmen und Ausgaben, Erträgen und Aufwendungen muss nicht nur im jeweiligen Haushaltsjahr, sondern auch in der Zukunft erreicht werden. Wird diese Vorgabe verfehlt, geht jeder kommunalpolitische Handlungsspielraum verloren. In einigen Ländern gibt es zahlreiche Beispiele für solche Folgen verfehlter Etatentscheidungen. Schließlich ist der Haushalt das effektivste Mittel zur Einflussnahme auf die hauptamtliche Verwaltung. Dieser Komplexität muss bei der wissenschaftlichen Diskussion über die Bürgerbeteiligung am kommunalen Haushalt Rechnung getragen werden.

Sieht man von einer knappen Einführung (S. 19-24) ab, ordnet Müller seine an der Humboldt-Universität zu Berlin entstandene und von Rosemarie Will betreute Dissertation dem Untertitel entsprechend in zwei Teile zu den Möglichkeiten der Beteiligung de lege lata (S. 25-192) und de lege ferenda (S. 193-277). Der erste Teil beginnt mit einem längeren Abschnitt über Elemente der direkten Demokratie in der Kommunalverfassung (S. 25-97). Hier stellt er die verfassungsrechtlichen Grundlagen in Art. 28 GG und den Landesverfassungen vor und gibt einen Überblick über die Beteiligungsformen in den Kommunalverfassungen. Im zweiten Abschnitt werden zuerst die auf Finanzfragen bezogenen kommunalverfassungsrechtlichen Ausschlusstatbestände für Bürgerbegehren und -entscheide dargestellt und auf ihre Reichweite untersucht (S. 97-140). Der Verfasser stützt sich dabei in erster Linie auf die Diskussion zur direkten Demokratie in den Ländern. Die „Besonderheiten der kommunalen Ebene“ nehmen dagegen nur bescheidenen Raum ein (S. 135-139). Den Auftakt des zweiten Abschnittes über informelle Formen der Bürgerbeteiligung in Finanzfragen (S. 140193) bilden knappe Ausführungen zu deren Rahmenbedingungen. Näher betrachtet und rechtlich bewertet werden dann die Beteiligung im Rahmen des Programms „Soziale Stadt“ am Beispiel der Berliner Quartierfonds (S. 146-152) und verschiedene Formen der Bürgerbeteiligung (S. 191). Beiden Modellen stehen keine rechtlichen Bedenken entgegen.

De lege ferenda wird im zweiten Teil die Möglichkeit geprüft, die Ausschlusstatbestände der Gemeindeordnungen für den Haushalt aufzuheben. Nach Auffassung Müllers könnte die Aufhebung die Möglichkeit eröffnen, die Bürger stärker in die Aufstellung des Haushaltsplanes einzubeziehen; ihnen die Änderung des Haushalts durch eine Nachtragshaushaltssatzung zu gestatten, hält er aus zeitlichen Gründen für nicht praktikabel (S. 194-201). Bevor er die verfassungsrechtliche Zulässigkeit einer solchen Änderung prüft, geht er auf die Rechtsprechung zu entsprechenden Vorschlägen für das Landesverfassungsrecht ein (S. 201-219). Zur Feststellung der Vereinbarkeit einer solchen Änderung des Kommunalverfassungsrechts mit dem Homogenitätsprinzip des Art. 28 Abs. 1 Satz 1 GG prüft er die Vereinbarkeit mit Art. 79 Abs. 3 GG, mit dem Demokratie- und dem Rechtsstaatsprinzip (S. 220-252). Als weitere grundgesetzliche 
Maßstäbe werden Art. 28 Abs. 1 Satz 2 und Art. 28 Abs. 2 sowie Art. 109 Abs. 2 GG herangezogen (S. 252-267). Den Abschluss bildet die Prüfung am Landesverfassungsrecht (S. 267-275); auch hier soweit vorhanden unter Einschluss der Ewigkeitsklauseln. Nach Auffassung des Verfassers steht Verfassungsrecht der Aufhebung der Ausschlusstatbestände in den Kommunalverfassungen nicht entgegen.

Die Arbeit hat ein weites Thema, das nicht präzise bestimmt ist. Die Untersuchung schwankt nämlich zwischen Finanzfragen, hierhin gehören auch Abgabenfragen und die Jahresrechnung (S. 102 ff.), und Haushaltsentscheidungen, auf die sich seine Zulässigkeitsdiskussion beschränkt. Sie gibt auf die Fragen, mit welchem Ziel die Bürgerbeteiligung hier ausgeweitet werden sollte, welche Möglichkeiten dafür bestehen, und wie sie ausgestaltet werden könnte, keine überzeugenden Antworten, sondern begnügt sich mit der Erörterung von Beispielen. Haushaltsspezifische Beteiligungsformen werden an einer Stelle kurz als historische Reminiszenz (S. 136), aber nicht als geltendes Recht ( $\S 80$ Abs. 3 GO NRW, 76 Abs. 1 GO Sachsen) erwähnt, zu dem Erfahrungen vorliegen. Auch zu der Frage, ob und mit welchen Ergebnissen der Bürgerantrag, der in vielen Ländern inhaltlich nicht beschränkt ist (S. 57), zu Haushalts- und Finanzfragen eingesetzt wird oder werden kann, findet sich nichts. Wieso Art. 79 Abs. 3 GG und die Ewigkeitsklauseln der Landesverfassungen, also Maßstabsnormen für Verfassungsänderungen, ausführlich als Maßstäbe für kommunalgesetzliche Regelungen diskutiert werden, erschließt sich nicht. Die Überlegungen zu den Maßstäben Demokratieprinzip und Selbstverwaltungsgarantie leiden darunter, dass Müller völlig darauf verzichtet, die Instrumente einer de lege ferenda möglichen Bürgerbeteiligung in den Rahmen der Bestimmungen über Haushaltsaufstellung und Haushaltsvollzug einzuordnen, und die eingangs erwähnten Restriktionen nur rudimentär erwähnt. Ohne eine nähere Diskussion der Ausgestaltungsfragen lassen sich auch über ein interessantes Instrument wie verbindliche Eckwertebeschlüsse (S. 199 f.) fundierte verfassungsrechtliche Aussagen nicht treffen. - Die Arbeit bietet die eine oder andere Anregung und Material etwa zum Thema Bürgerhaushalt; die Diskussion über Bürgerbeteiligung in Haushalts- und Finanzfragen der Kommunen steht aber immer noch am Anfang.

Janbernd Oebbecke

Volker Mittendorf: Die Qualität kollektiver Entscheidungen. Kommunikationsprozesse direkter und repräsentativer Demokratie im Vergleich, Campus Verlag: Frankfurt/New York 2009, 285 S., 34,90 Euro.

Dem anzuzeigenden Werk liegt eine an der Universität Marburg bei Theo Schiller entstandene politikwissenschaftliche Dissertation zugrunde, die der Frage 
nachgeht, ob direktdemokratische Verfahren sich auf die „Handlungskultur der Akteure und den Entscheidungshintergrund“" auswirken. Die etwas sperrig anmutende Fragestellung wird durch fünf weitere Fragen ausdifferenziert (20), namentlich: [a] welche Auswirkungen direktdemokratische Verfahren auf die Berücksichtigung von Grund- und Menschenrechten in der politischen Handlungspraxis haben; [b] wie sich Abstimmungen auf Partizipationsansprüche von Beteiligten auswirken; [c] welche Auswirkungen direktdemokratische Verfahren auf die Wahrung der politischen Gleichheit haben; [d] wie sich Wahlen und Abstimmungen mit Blick auf die formulierten Argumente und Entscheidungsgründe unterscheiden; [e] wie die Auswirkungen der direktdemokratischen Entscheidungen im Vergleich zu Wahlen wahrgenommen werden. Insgesamt kann das Werk als Beitrag zur Erforschung der funktionalen Gemeinsamkeiten und Unterschiede von Wahlen und Abstimmungen verstanden werden. Dabei werden Abstimmungen pragmatisch als Verfahren zur Legitimation und Wahlen als Verfahren zur Legitimation von Legitimation verstanden (25).

Das Buch lässt sich grob in drei Teile gliedern: Der erste Teil (Kapitel 1 bis 6, 13-111) dient der Begriffsbildung und der theoretischen Grundlegung, mit der der Verf. seine kommunikationszentrierte Konzeption des Geflechts von Demokratie, Repräsentation, Wahlen und Abstimmungen darlegt. Im zweiten Teil leitet der Verf. aus seiner Konzeption Hypothesen ab (Kapitel 7, 113-121) und erläutert in einem knappen methodischen Abschnitt (Kapitel 8, 123-129), wie er diese operationalisieren und empirisch überprüfen will. Der dritte Teil (Kapitel 9-12, 131-250) dient schließlich eben dieser empirischen Überprüfung anhand von insgesamt 18 Beobachtungen (je neun Wahlen und Abstimmungen) in drei Städten (Luzern, Marburg und St. Gallen).

$\mathrm{Zu}$ Beginn (2. Kapitel) konturiert der Verf. die Begriffe „Demokratie“ und „Repräsentation“ wie er sie für die weitere Arbeit zugrunde legen will. Wesentlicher Anknüpfungspunkt für die Begriffsbildung ist die mindestens notwendige Anzahl unterschiedlicher Beteiligter, was zur Einordnung der Demokratie als zweistelliger Prädikator (Autor der Herrschaftsbeziehung $\rightarrow$ Adressat der Herrschaftsbeziehung, 29) und der Repräsentation als dreistelliger Prädikator (Bezugsobjekt $\rightarrow$ Repräsentant $\rightarrow$ Adressat, 34) führt. Wenig überzeugend sind die sehr holzschnittartigen historischen Ausführungen, die angesichts ihres beschränkten Umfangs zur Begriffsbildung nicht beitragen, sondern eher Widerspruch provozieren. Bei der Entwicklung der Begrifflichkeit zur Repräsentation vermisst man die Bedeutungsdimension der „Abbildung“, wie sie etwa dem Leibholz'schen Repräsentationsverständnis zugrunde lag, demzufolge die Idee des Parlaments in der liberalen Demokratie die Abbildung des Gesamtvolkes sei (er verwendet den Begriff also, wie er heutzutage verwendet wird, wenn man davon spricht, eine empirische Stichprobe sei „repräsentativ“ für die Gesamtpopulation). Ein solches Begriffsverständnis hätte sich freilich auch nicht als drei- 
stelliger Prädikator qualifizieren lassen. Außerdem fällt auf, dass Hasso Hofmanns maßstabsetzende Schrift Repräsentation nur unzureichend rezipiert worden ist. Gewinnbringend sind die von der Inkongruenz der Begriffe Demokratie und Repräsentation ausgehenden weiteren Überlegungen zum Verhältnis der beiden Konzepte (39 ff.).

Im nächsten Kapitel widmet sich der Verf. der Frage, ob direktdemokratische Verfahren zur „Qualifizierung von Demokratie“ über ein „Normal- bzw. Mindestmaß der Qualifikation einer Demokratie als repräsentative Demokratie hinaus“ (45) beitragen können. Dabei geht er im Anschluss an die bestehende Literatur (v.a. Theo Schiller) vom Qualifizierungsansatz aus (45 und 46 ff.), demzufolge über die Grenzen verschiedener Demokratietheorien hinweg gewisse Kernelemente zu identifizieren sind, welche eine Staatsform zumindest basal aufweisen muss, um als Demokratie qualifiziert werden zu können (und in denen die eingangs formulierten Forschungsfragen einen gewissen Widerhall finden), namentlich: [1] Grund- und Menschenrechte (vgl. oben [a]), [2] Offenheit der Machtstruktur (vgl. oben [b]), [3] politische Gleichheit (vgl. oben [c]), [4] Transparenz und Rationalität (vgl. oben [d]) und [5] politische Effektivität (vgl. oben [e]). Daneben flössen auch empirische (d.h. kognitiv einsehbare) und normative (d.h. moralisch wertende) Komponenten in den Entscheidungsprozess ein, die den politischen Prozess und seinen Output demokratisch qualifizierten (Wissensqualität und sozial-moralische Qualität, $51 \mathrm{f}$.). Schließlich könne ggf. auch die Öffentlichkeit durch die indirekten Wirkungen von Volksabstimmungen qualifiziert werden (52 f.). In welchem Verhältnis die Qualifizierungsmodi zu einander stehen und wie dadurch „Input und Output von Demokratie aufeinander bezogen betrachtet werden“ (51) können, bleibt offen.

Um beurteilen zu können, ob und wie es in Wahlen und Abstimmungen zu einer Qualifizierung der Öffentlichkeit kommen kann, widmet sich der Verf. sodann der Frage, wie das „Volk“ als Kollektivakteur überhaupt zu konkreten Handlungen und insbesondere zu Herrschaft fähig sein soll (Kapitel 4). Als Lösung bietet er „kollektive Aggregationshandlungen“ (58) an, die vor der Folie einer Theorie kommunikativen Handelns (59 ff.) nach vorheriger Verständigung der Handlungsbeteiligten - obwohl auf ein Individuum zurückführbar - als vom Volk getätigte verstanden werden sollen (eine Art Zurechnung also); mindestens Wahl und Abstimmung seien als tatsächliche kollektive Handlungen zu verstehen. Herrschaft sei danach eine besondere Form der Kommunikation (60). Als Kommunikationsmodi, die zur Herstellung verbindlicher Entscheidungen genutzt werden können, benennt der Verf. „Argueing“ (als Kommunikationsform im Sinne von Habermas' Theorie kommunikativen Handelns), „Bargaining“ (als der Rationaltheorie verpflichtete Form der Entscheidungsfindung), „Akklamation“ bzw. „Konsensfeststellung“ (als plebiszitäre Führerkonzepte) und „Aggregation“ (als Mechanismus nach der Theorie sozialer Wahlhandlungen). Auch hier ist 
nicht klar, in welchem Verhältnis die Kommunikationsmodi zueinander stehen, und die beunruhigende Frage, ob „kollektive Aggregationshandlungen“ (auch wenn Kommunikationsverfahren gewählt werden, die nicht zum AggregationsModus zu zählen sind) nicht auch jenen Aggregationsproblemen unterliegen, die grundlegend etwa Arrow beschrieben hat (und wenn nicht: was uns die Erlösung verschafft), bleibt gänzlich unbehandelt. Insofern bleiben auch die Ergebnisse zu diesem Fragenkomplex insgesamt vage und unbefriedigend (69).

In den Kapiteln 5 und 6 (71-111) gibt der Verf. einen instruktiven Überblick über den Stand der Wahl- und Abstimmungsforschung; inwiefern diese Literaturschau dazu beiträgt, die Fragestellung des Werkes abzuarbeiten, wird nicht immer deutlich. In Kapitel 7 stellt der Verf. schließlich auf der Basis von sechs Annahmen insgesamt acht Hypothesen auf, die er vier der fünf o.g. Qualifizierungsmerkmalen von Demokratie zuordnet:

\section{(Offenheit der Machtstruktur)}

1. In Abstimmungen treten andere Akteure auf als in Wahlen.

2. In Abstimmungskämpfen treten mehr politikfeldspezifische Akteure auf als zum gleichen Thema in einem Wahlkampf.

3. In Abstimmungen verbinden sich Akteure, die bei Wahlen getrennt voneinander auftreten.

\section{(Politische Gleichheit)}

4. In Wahlen und bei kontroversen Abstimmungen wird über alle Entscheidungsalternativen in gleichem Umfang berichtet.

\section{(Transparenz und Rationalität)}

5. In Wahlen geht es stärker um Personen- und Gruppenkompetenz und um die Priorisierung von Themen, bei Abstimmungen stehen verstärkt Sachfragen, Ursachen- und Wirkungszusammenhänge und die Eignung von Maßnahmen im Fokus.

6. In Abstimmungen kommt es zu einer höheren Validierung von Aussagen, bei Wahlen werden Probleme transparenter definiert; Orientierungsaussagen kommt bei beiden Verfahren der gleiche Stellenwert zu.

\section{(Politische Effektivität)}

7. Bei Wahlen und Abstimmungen wird gleichermaßen Bezug genommen auf den Einfluss zuvor getroffener Entscheidungen für die anliegende Entscheidung und die Auswirkungen der projektierten auf zukünftige Entscheidungen. 
8. Die (politische) Effektivität steigt mit der Anzahl der Abstimmungen.

Im achten Kapitel beschreibt der Verf. auf (im Verhältnis zu den vorangegangenen Ausführungen, aber den Gepflogenheiten der deutschsprachigen Politikwissenschaft wohl entsprechend: nur) sechseinhalb Seiten Methode und Untersuchungsdesign, mit der er diese Hypothesen empirisch testen möchte. Er wählt einen most-similar-systems-Ansatz, der zur Klasse der quasi-experimentellen zu zählen ist (124). Für den zwar mit sozialwissenschaftlichen (auch empirischen) Methoden, nicht aber mit den Details der politikwissenschaftlichen Terminologie vertrauten Leser sind die 14 Zeilen zum Untersuchungsdesign unergiebig. So wird beispielsweise erst im Verlauf der empirischen Untersuchung klar, dass der Verf. einen qualitativ orientierten quasi-experimentellen Ansatz wählt (während die meisten entsprechenden Studien quantitativ vorgehen, epochal etwa Sloan et al. 1988). Der most-similar-systems-Ansatz dient im Rahmen der Fallstudie der Begründung der Auswahl des Fallmaterials. Diese Begründung - die auf der Vergleichbarkeit der Städte Luzern, Marburg und St. Gallen fußt - fällt ebenfalls knapp aus, ist aber intuitiv überzeugend.

In Kapitel 9 beschreibt der Verf. detailliert die ausgewählten Fälle, zunächst mit Blick auf die in den drei Städten zwischen 1991 und 2001 abgehaltenen Wahlen sowie die Charakteristika der an ihnen beteiligten Gruppen (Parteien), Personen und der verhandelten Sachthemen. Kapitel 10 fokussiert sich dann auf das Politikfeld Verkehr und schildert kenntnisreich die Hintergründe, das Berichtsumfeld sowie die Charakteristika der Gruppen, Personen und Sachthemen, die Beteiligte bzw. Gegenstand von Abstimmungen waren. In Kapitel 11 schließlich analysiert der Verf. anhand einer Auswertung aller in einem bestimmten Zeitraum um die insgesamt 18 Wahlen und Abstimmungen in den verschiedenen Orten jeweils zur Verfügung stehenden lokalen Tageszeitungen (redaktionelle Beiträge, Anzeigen, Leserbriefe), die Entwicklung der Entscheidungskommunikation (209). Hier wird auch das methodische Vorgehen eingehend erklärt.

Nach der deskriptiven Analyse der so gewonnen Daten wendet sich der Verf. den acht Hypothesen zu den vier Fragestellungen seiner Arbeit zu (219 ff.). Die ersten beiden Hypothesen - dass bei Abstimmungen andere und mehr Akteure auftreten als bei Wahlen - findet er (jeweils durch die methodisch einwandfreie Verwerfung der gebildeten gegenteiligen Null-Hypothese) in seinen Daten bestätigt und folgert daraus eine Öffnung der Machtstruktur bzw. eine Erhöhung der Transparenz (225 f.). Aus der intimen Kenntnis der beobachteten Fälle kann er sogar eine Reihe möglicher Ursachen herausarbeiten. Die Evidenz zur dritten Hypothese - dass sich bei Abstimmungen Koalitionen von Akteuren bilden, die bei Wahlen getrennt voneinander auftreten - ist nicht eindeutig, scheint aber ebenfalls in Richtung einer Öffnung der Machtstrukturen zu deuten, vor allem für die beiden Schweizer Städte (229). Hypothese 4 - in Wahlen und Abstim- 
mungen wird in vergleichbarem Umfang über alle Entscheidungsalternativen berichtet - sieht der Verf. durch die beobachteten Berichtsmuster mit Ausnahme punktueller Verzerrungen ebenfalls bestätigt (230 f.). Auch Hypothese 5 - Personen- und Prioritätsfokus bei Wahlen, Sach- und Maßnahmeneignungsfokus bei Abstimmungen - wird durch die beobachteten Fälle, wenig überraschend, bestätigt (233 ff.). Zur sechsten Hypothese - höhere Aussagenvalidierung bei Abstimmungen, höhere Transparenz der Problemdefinitionen bei Wahlen, vergleichbarer Stellenwert von Orientierungsaussagen bei Wahlen und Abstimmungen - liefert der Verf. nur eine Beschreibung des Datenmaterials, formuliert aber kein Ergebnis (235 ff.). Die Daten zu Hypothese 7 - Bedeutung vergangener Entscheidungen für die jetzige und der jetzigen für zukünftige - lassen ebenfalls keine eindeutigen Aussagen zu, sind aber insofern interessant, als sie nahelegen, dass die Frage, ob es sich um eine Wahl oder eine Abstimmung handelt (Variable „Ereignisbezug“), hier keinen entscheidenden Einfluss hat (238). Für die letzte Hypothese - dass die politische Effektivität von Abstimmungen mit der Anzahl der Abstimmungen steige - lassen sich ebenfalls Anhaltspunkte finden, die aber entlang der Institutionengrenze Schweiz/Deutschland verlaufen und insofern weiterer Untersuchung bedürften.

Wenngleich die Ergebnisse angesichts des präsentierten Materials plausibel erscheinen, wäre es wünschenswert gewesen, die Effekte zu quantifizieren, um die qualitative fundierte Aussage weiter abzustützen. Sowohl die Fragestellungen als auch die vorhandenen Daten hätten sich für eine zusätzliche quantitative Evaluation gut geeignet. Dass der Verf. sich dieser Mühe nicht unterzogen hat, ist bedauerlich, zumal die Daten erhoben und sogar codiert waren, eine quantitative Auswertung also möglich gewesen wäre. Das hätte es dem Verf. schließlich auch ermöglicht, klarere Ergebnisse zu formulieren, anstatt, wie in diesem für die Arbeit entscheidenden Kapitel, im wesentlichen die Beobachtungen zu schildern, die Annahme oder Verwerfung der Hypothese dann aber letztlich doch dem Leser zu überlassen.

Insgesamt handelt es sich um eine lesenswerte Studie, in der man anhand von instruktivem und kenntnisreich vorgetragenem Anschauungsmaterial einiges über Gemeinsamkeiten und Unterschiede von Wahlen und Abstimmungen lernen kann. Der Verfasser nimmt dafür eine beachtliche Anzahl unterschiedlicher Diskussions- und Literaturstränge auf; das ist einerseits eine Stärke der Arbeit, macht sie aber auch ein Stück weit unübersichtlich, lang und schwer lesbar. Für den explorativen Zweck der Arbeit, für ein Herantasten auf dieses Feld, scheint das aber ein sinnvoller Zugang zu sein. Die Arbeit leistet einen wichtigen Beitrag dazu, die Tür zu den dort aufgeworfenen Fragen ein Stück weit aufzustoßen.

Emanuel V. Towfigh 
